\title{
A Reliable and Efficient Time Synchronization Protocol for Heterogeneous Wireless Sensor Network
}

\author{
Masoume Jabbarifar, Alireza Shameli Sendi, Alireza Sadighian, \\ Naser Ezzati Jivan, Michel Dagenais \\ École Polytechnique de Montréal, Montreal, Canada \\ E-mail: \{masoume.jabbarifar, alireza.shameli-sendi, alireza.sadighian, naser.ezzati-jivan, \\ michel.dagenais\}@polymtl.ca \\ Received October 16, 2010; revised November 1, 2010; accepted November 8, 2010
}

\begin{abstract}
L-SYNC is a synchronization protocol for Wireless Sensor Networks which is based on larger degree clustering providing efficiency in homogeneous topologies. In L-SYNC, the effectiveness of the routing algorithm for the synchronization precision of two remote nodes was considered. Clustering in L-SYNC is according to larger degree techniques. These techniques reduce cluster overlapping, resulting in the routing algorithm requiring fewer hops to move from one cluster to another remote cluster. Even though L-SYNC offers higher precision compared to other algorithms, it does not support heterogeneous topologies and its synchronization algorithm can be influenced by unreliable data. In this paper, we present the L-SYNCng (L-SYNC next generation) protocol, working in heterogeneous topologies. Our proposed protocol is scalable in unreliable and noisy environments. Simulation results illustrate that L-SYNCng has better precision in synchronization and scalability.
\end{abstract}

Keywords: Wireless Sensor Network, Synchronization, Clustering, Heterogeneous, Convex Hull

\section{Introduction}

In recent years, wireless sensor networks have been used in wide range of applications including oil industry, medical, and military services. They can be used in such environments to collect data from movements of objects, to measure the speed and flow direction of oil spills, or to control and track goods in a warehouse. Clustering can be used in wireless sensor networks to implement these networks and has some advantages such as extending the lifetime of the network, decreasing consumption of energy, reducing routing overhead, and calculating route path. Selecting less overlapped clusters in wireless sensor networks results in better performance for high level network functions such as routing, query processing, data aggregation and broadcasting [1]. In the past few years, several algorithms have been suggested for time synchronization of sensor networks. In this paper, we propose a time synchronization protocol for heterogeneous and homogeneous sensor network topologies. As we use the convex hull synchronization algorithm between sensors, the result is better efficiency in unreliable noisy environments.

The rest of the paper is organized as follows: in Section
2, we investigate earlier work and several clustering methods. Comparison between convex hull and regression techniques will be presented in Section 3. In Section 4, the proposed protocol will be discussed. Experimental results are given in Section 5. The last section concludes this paper by outlining future work that will follow.

\section{Related Work}

In this section, we mention some recent synchronization algorithms and present an overview on common clustering methods. Generally, time synchronization protocols are categorized into two main techniques: 1) Synthetic: In this technique, time estimations are done several times to get the local time of a node and eventually generate a function for each node. The more data, the more precise approximations. 2) Non-synthetic: In this technique, a sample of time estimation (less overhead) is considered as the foundation of synchronization. Essentially, this technique is faster but less precise than the synthetic technique. Table 1 shows these techniques and the related algorithms [2].

In the following, we explain the prevalent time synchronization protocols which have used the mentioned 
Table 1. Time synchronization techniques.

\begin{tabular}{ll}
\hline \multicolumn{1}{c}{ Synthetic techniques } & \multicolumn{1}{c}{ Non- synthetic techniques } \\
\hline - Linear regression & - Unidirectional Synchronization \\
- Phase-locked loops & - RoundTrip Synchronization \\
& - Reference Broadcasting \\
& - Pair-Wise \\
\hline
\end{tabular}

techniques. Table 2 also categorizes these protocols in terms of network topology and synchronization techniques.

- RBS: In this protocol, non-synthesized Reference Broadcasting method is used to compute the difference between nodes' offset [3]. According to nonsynchronous clock ticks, linear regression is used such that each node determines the best fitted line from its local time and its neighbor local time. Slope of this line is the velocity of clock changes.

Table 2. Classification of time synchronization protocols.

\begin{tabular}{|c|c|c|}
\hline Algorithm & Network topology & Synchronization technique \\
\hline RBS [3] & $\begin{array}{l}\text { Several reference nodes and } \\
\text { synchronization in each } \\
\text { reference domain }\end{array}$ & $\begin{array}{l}\text {-Reference Broadcast } \\
\text {-Linear regression }\end{array}$ \\
\hline LTS [4] & $\begin{array}{l}\text { Spanning tree with low } \\
\text { depth (first depth search) }\end{array}$ & $\begin{array}{l}\text { Pair-Wise (from root to } \\
\text { children) }\end{array}$ \\
\hline TPSN [5] & Hierarchical-tree structure & $\begin{array}{l}\text { Pair-Wise (level i with level } \\
\text { i-1) }\end{array}$ \\
\hline T-sych [6] & $\begin{array}{l}\text { Tree structure; many refer- } \\
\text { ence nodes }\end{array}$ & $\begin{array}{l}\text {-HRTS: with Pair-Wise but } \\
\text { in broadcasting method } \\
\text {-ITR: two nodes sync. inde- } \\
\text { pendently }\end{array}$ \\
\hline PCTS [7] & Clustering based on ID & Averaging by cluster head \\
\hline CHTS [8] & $\begin{array}{l}\text {-Clustering based on ID } \\
\text {-Tree formation between } \\
\text { cluster heads and member } \\
\text { nodes }\end{array}$ & $\begin{array}{l}\text { Cluster heads will be syn- } \\
\text { chronized with reference } \\
\text { node by Pair-Wise method }\end{array}$ \\
\hline $\mathrm{AD}[9]$ & No structure & Averaging by each node \\
\hline FTSP [10] & $\begin{array}{l}\text { Less node ID is reference } \\
\text { node }\end{array}$ & $\begin{array}{l}\text { Linear regression by nodes } \\
\text { after sending } 8 \text { times by } \\
\text { reference node }\end{array}$ \\
\hline SLTP [11] & $\begin{array}{l}\text { Clustering based on ID } \\
\text { technique }\end{array}$ & $\begin{array}{l}\text { Linear regression after } \\
\text { sending } 10 \text { times by cluster } \\
\text { head }\end{array}$ \\
\hline $\begin{array}{l}\text { L-SYNC } \\
{[12]}\end{array}$ & $\begin{array}{l}\text { Clustering based on degree } \\
\text { technique }\end{array}$ & $\begin{array}{l}\text { Linear regression after } \\
\text { sending } 10 \text { times by cluster } \\
\text { head }\end{array}$ \\
\hline
\end{tabular}

- PCTS: This protocol uses ID-based method (passive) and considers node clustering [7]. Cluster head alternatively gathers local clocks of its cluster members and computes the average. Afterward, the cluster head will broadcast the average time.

- CHTS: In this protocol, nodes can change their radio domain [8]. Some of the nodes are high performance nodes and others are low performance nodes. This protocol also uses ID-based method for clustering the nodes. Cluster heads are selected among high performance nodes. In this protocol, firstly cluster heads are synchronized in Pair-Wise technique with reference node and then cluster head will announce the time to all cluster members.

- SLTP: This protocol uses ID-based method for node clustering [11]. The cluster head sends its local time continuously to cluster members at specified time intervals. Using linear regression method, cluster members calculate time interval and velocity changes of its clock with cluster head clock. SLTP method is same as RBS in precision; however for wide areas and long life clusters, SLTP operates more efficiently.

Three criteria are used to select cluster head $(\mathrm{CH})$ :

1) ID-based method: This method assigns a unique ID to each node. One strategy is the selection of nodes having lower ID as the cluster head.

2) Degree-based method: In this method (the degree of a node is the number of its neighbors) nodes with higher degrees can be selected as cluster head [13]. These methods attempt to minimize the number of cluster heads, minimizing clusters overlap. Fewer clusters and overlaps will decrease the channel competition between clusters and also will improve the algorithm efficiency [1].

3) Weight-based method: In this method, several parameters may be considered for $\mathrm{CH}$ selection. These parameters include remaining energy, degree, dynamicity, and average distance to neighbors $[10,14]$.

\section{Convex Hull vs. Linear Regression}

When a message is exchanged between a pair of nodes, the receiving and sending times will not be reliably comparable because the clocks of two nodes are not synchronized. By the principle of causality, the received time must be after the sent time. This constraint is used to compute the clock drift between two nodes.

Two proposed synchronization algorithms are Linear Regression and Convex Hull [15]. Both algorithms try to estimate a linear conversion function between the clocks in a pair of nodes. The drift and offset of the two clocks are extracted from linear function. In a two dimensional space, based on timestamps of node A and B, the Linear 
Regression algorithm tries to find a fitted line among points. Each point impacts the position of the fitted line. In the synchronization process, network latency and related problems between two nodes cause erratic, delayed, time values. Ideally, these points should not influence the fitted line and they should be ignored in the calculation to increase synchronization accuracy. The Convex Hull is an algorithm that assumes minimum sent timestamps and maximum received timestamps. It finds the area that has minimum latency and ignores far points. Hence the estimated line is more accurate than Linear Regression.

\section{Proposed Method}

In our proposed method, the synchronization is performed between cluster members and the cluster head. It is not necessary for cluster members to exchange and analyze synchronization data. However, each pair of nodes (within the same cluster or even in two different clusters) may be synchronized if needed. The synchronization is not affected by the fact that nodes may differ in strength, ability and radio domain. Each node is able to change its role from cluster head to cluster member and vice-versa. The proposed algorithm does not change the clock time of the nodes, instead the clock offset and clock skew of each node will be calculated with respect to cluster head clock. To compare synchronization accuracy, nodes local clock in different clusters are compared together. The proposed algorithm proceeds in two phases: configuration and synchronization. We explain these phases in the next section. Figure 1 illustrates the pseudo-code of L-SYNCng protocol.

\subsection{Configuration Phase}

As mentioned, the SLTP protocol uses passive clustering method for homogeneous and heterogeneous topologies [11]. L-SYNC is only efficient in homogeneous environments. In this work, we have applied some strong clustering methods such as DCA (weight-based) and ACE (degree-based) to our model (L-SYNCng) in order to address the L-SYNC shortcomings in heterogeneous distributions. After investigation of the mentioned clustering methods, we have retained the method providing better results. We explain the DCA and ACE clustering methods in the following.

The ACE algorithm [13] is based on adjacency degree. This algorithm results in highly uniform clustering and achieves an efficient cluster topology, nearly hexagonal. Using self-organizing characteristics within clusters, the algorithm creates well-separated clusters. Indeed, results show that clusters are less overlapping than other algorithms. ACE has two steps, spawning new clusters and migrating existing clusters. To prevent collisions, each node chooses a random interval. This algorithm is iterative. When a node's turn comes, it starts processing to determine its role. At the beginning, each node is in the unclustered condition, so each node starts to calculate its number based on loyal followers (identified as $l$ ). A loyal follower is a neighbor which is the member of at most one cluster. In the initiation phase, this number is equal to the number of unclustered neighbors of a node. As clustering proceeds, each node, for example node $\mathrm{A}$, knows the time elapsed since the beginning of the protocol (identified as $t$ ). Afterward, node A starts to compute the cluster spawning threshold function $f_{\min (t)}$.

If $l>f_{\min (t)}$, node A can spawn a new cluster. Each node executes the protocol at least for $C I$ time, where $C$ is the desired average number of iterations per node

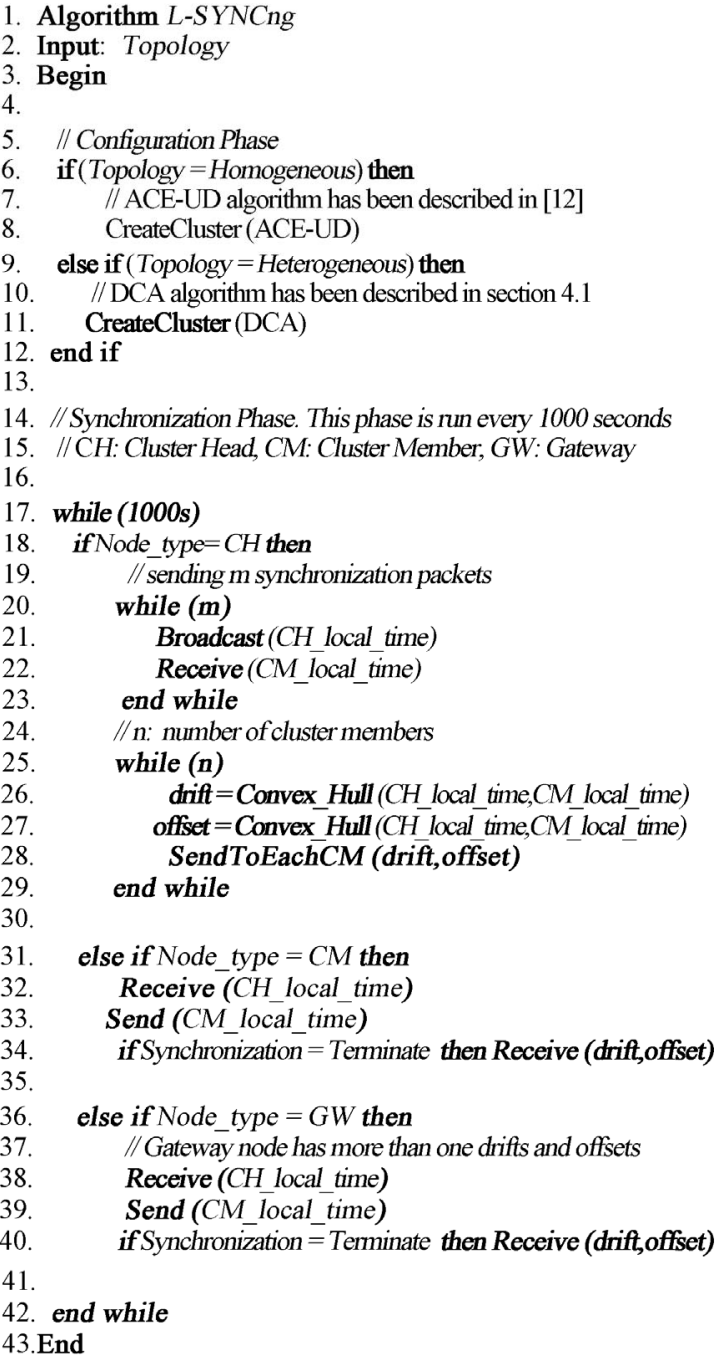

Figure 1. Pseudo-code of L-SYNCng Protocol. 
and $I$ is the expected length of each iteration. $f_{\min (t)}$ function is an inverse exponential. At the beginning, it is equal to the average number of neighbors in the graph. The equation is as follows:

$$
f_{\min }|t|=\left(e^{-k_{1} t C I}-k_{2}\right) d
$$

In this equation, $t$ is the elapsed time from the start of the protocol, $C I$ is the duration of the protocol, $d$ represents the average number of neighbors in the network. This average is computed in a pre-processing step. $\mathrm{K}_{1}$ and $\mathrm{K}_{2}$ are constants determining the shape of the exponential function.

The algorithm designers have selected empirically $\mathrm{K}_{1}$ $=2.3$ and $\mathrm{K}_{2}=0.1$ to obtain a good compromise between the clustering quality and the execution time. Using these values, $f_{\min }$ starts at $0.9 \mathrm{~d}$ and decreases to zero on the last iteration. This insures that each unclustered node chooses itself as a cluster head at the end of the protocol. When a node is already a cluster head, at the following iterations it checks whether neighbor nodes are better candidates as cluster head. The node polls all its neighbors to find the best candidate for being cluster head. Therefore, it sends a POLL message to all neighbors. The best candidate is the one containing the largest potential number of loyal followers in its neighbors' set. It means that each node which receives a POLL message starts counting neighbors that are unclustered or are only part of the cluster headed by node A. By counting loyal followers except nodes that are in two or more overlapping clusters, the best candidate node generally provides the least overlap with other clusters. If the best candidate to become cluster head is node $\mathrm{A}$, then A does nothing. If the best candidate is another node $(\mathrm{B}), \mathrm{A}$ migrates to the new cluster head $B$. A performs this migration by propagating a PROMOTE message to node $\mathrm{B}$. When receiving the PROMOTE message, $B$ propagates a RECRUIT message to form a cluster with A's cluster ID.

The Distributed Clustering Algorithm (DCA) [16] is a weight-based algorithm. In this approach, one node decides to become $\mathrm{CH}$ or join a cluster depending on information from its neighbors that are in one hop distance. This technique is essentially iterative.

In iterative clustering techniques, a series of nodes wait for a specific event, and other nodes decide their own role (for instance to become $\mathrm{CH}$ or not). In DCA, before deciding, one node waits until all its neighbors which have more weight make their decision, and change to $\mathrm{CH}$ or join existing clusters. Nodes that have largest weight among neighbors with one hop distance will be selected as $\mathrm{CH}$. A problematic issue in most iterative approaches is that convergence speed is dependent on network diameter (the path that includes the largest number of hops).
In a two dimensional field with $n$ distributed nodes, the DCA algorithm needs $O(\sqrt{n})$ iterations to finalize the solution. Generally, probabilistic approaches for clustering insure rapid convergence and provide desirable features such as balanced cluster size. This approach causes activation of each node independently to decide for its role within a clustered network, while keeping the message overhead low [13].

In the following, we explain how the mentioned clustering methods are used in L-SYNCng under heterogeneous topologies. Figures 2-3 illustrate the execution of ACE and DCA algorithms for 100 nodes distributed randomly (the path between nodes 0 and 99 is considered). As Figures 2 illustrates, after execution of ACE algorithm, clusters contain less overlap. In this execution, 13 nodes are selected as cluster heads. Once clustering has been done, routing algorithm was executed to find a route from node 99 to node 0 .

The routing path is as follows: CM (99) -> CH (78) -> GW (66) -> CH (45) -> GW (25) -> CH (14) -> GW (3) -> CH (2) -> CM (0). It means that to compare the time stamp of nodes 0 and 99, 8 hops are required with the conversion of time information based on Equation (2).

After execution of the DCA algorithm, as Figure 3 shows, 14 nodes are selected as cluster heads. Once clustering has been done, the routing algorithm was executed to find a route from node 99 to node 0 .

The routing path is as follows: CM (99) -> CH (78) -> GW (66) -> CH (45) -> GW (34) -> CH (32) -> GW (22) $->\mathrm{CH}(2)->\mathrm{CM}(0)$.

To compare the times of nodes 0 and 99, 8 hops are required where the conversion of time stamp information based on equation 2 takes place. Results of executing the two methods passing through hops from node 99 to node 0 are shown in Table 3.

As the results of DCA algorithm illustrate, the number of hops remains the same. As this algorithm needs fewer time conversions than ACE, better accuracy can be achieved.

Based on the above comparison, we can conclude that DCA is a better clustering algorithm for heterogeneous

Table 3. Comparison of hops from node 99 to node 0 in ACE and DCA techniques.

\begin{tabular}{ccc}
\hline Execution & ACE & DCA \\
\hline 1 & 9 & 8 \\
2 & 10 & 8 \\
3 & 8 & 8 \\
4 & 8 & 8 \\
5 & 9 & 8 \\
6 & 11 & 8 \\
7 & 8 & 8 \\
8 & 12 & 8 \\
9 & 10 & 8 \\
10 & 9 & 8 \\
Average & 9.4 & 8 \\
\hline
\end{tabular}




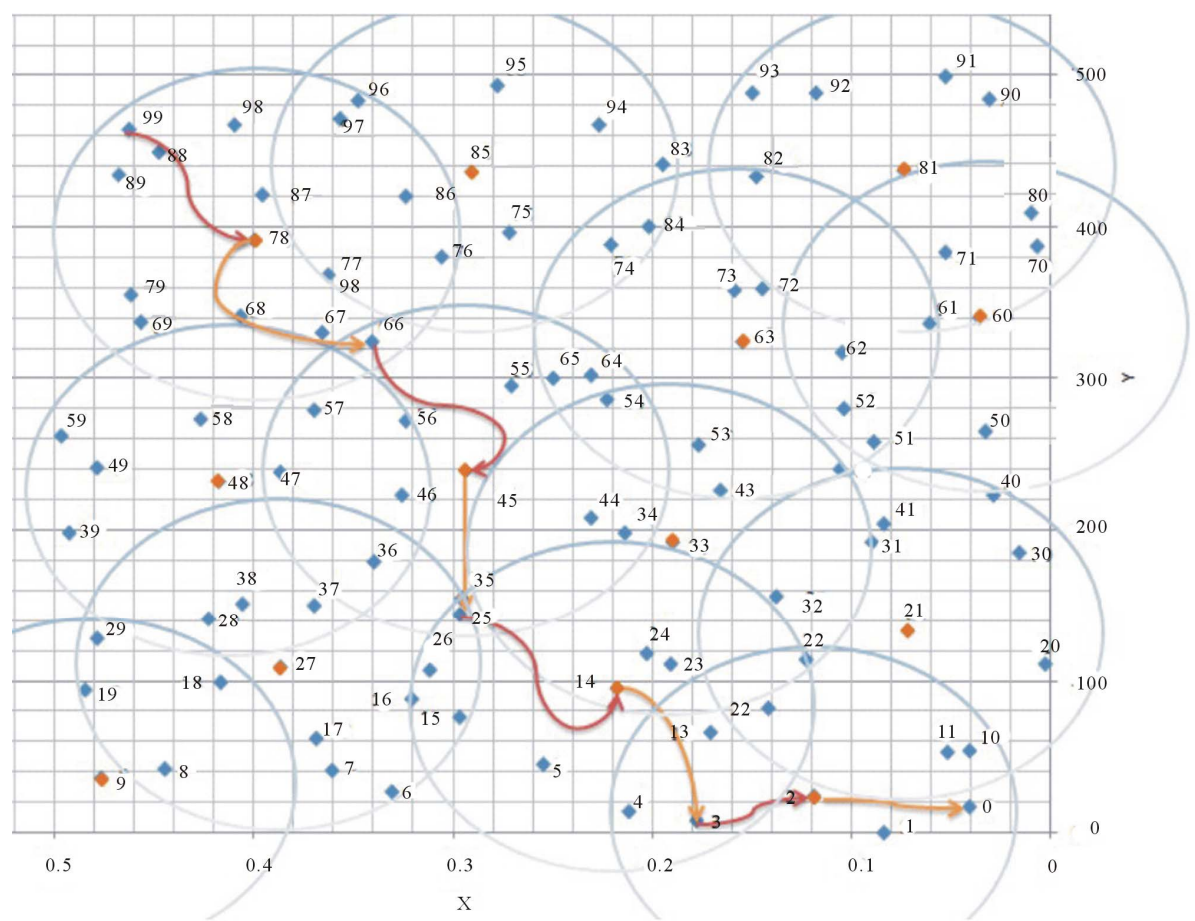

Figure 2. Using ACE as a clustering in L-SYNCng.

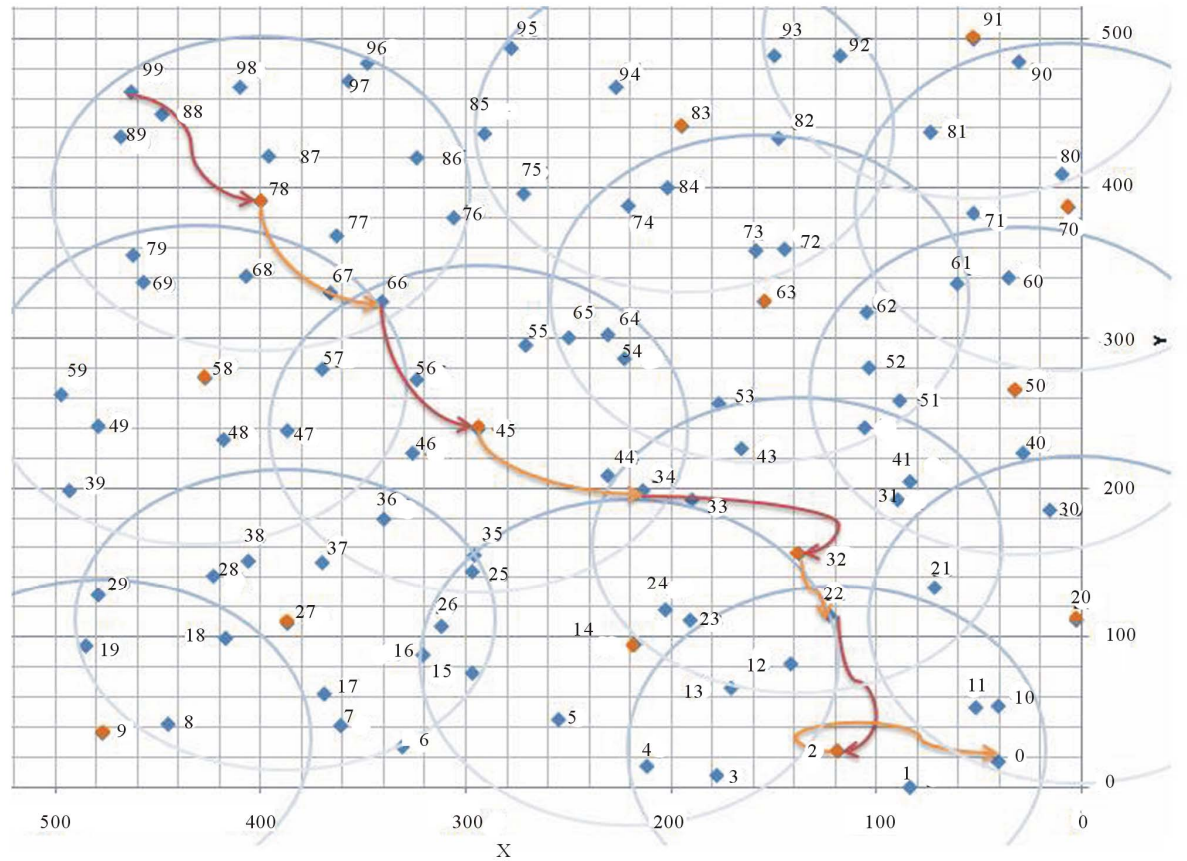

Figure 3. Using DCA as a clustering in L-SYNCng.

topologies. Therefore, this algorithm can be used by L-SYNCng in heterogeneous topologies.

\subsection{Synchronization Phase}

In this phase, each cluster head starts to broadcast a synchronization packet including its identity number and local time. Each cluster member receives this packet and sends an acknowledgment to the cluster head.

The cluster head waits to receive all ACK messages, as shown in Figure 4. Thus four timestamps $\left(t_{\mathrm{cs}}, \mathrm{t}_{\mathrm{mr}}, \mathrm{t}_{\mathrm{ms}}\right.$, $t_{c r}$ ) are generated for each ACK. If a cluster member re- 


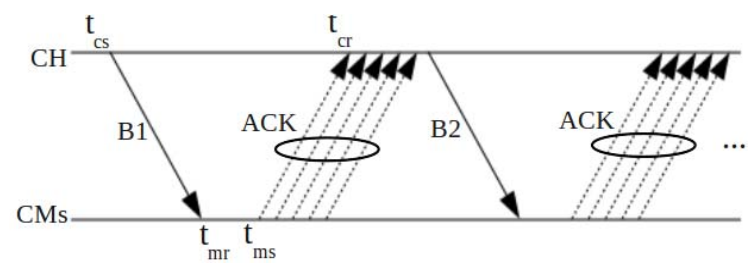

Figure 4. Synchronization packets between $\mathrm{CH}$ and CMs. CH starts synchronization.

plies immediately to the cluster head, $\mathrm{t}_{\mathrm{mr}}$ would be equal to $t_{\mathrm{ms}}$. A cluster member can delay as long as it wants. The precision will decrease if the delay between $t_{\mathrm{cs}}$ and $\mathrm{t}_{\mathrm{cr}}$ increases. Cluster head has $\mathrm{t}_{\mathrm{cs}}, \mathrm{t}_{\mathrm{ms}}$ and $\mathrm{t}_{\mathrm{cr} \text {. }}$

To determine $t_{m r}$ it is enough to have the minimum delay between $t_{\mathrm{mr}}$ and $t_{\mathrm{ms}}$. The minimum delay can be estimated by the cluster head for each cluster member at the end of broadcasting, based on the history of each cluster member's behavior [17].

The cluster head will broadcast a next synchronization packet. After sending $m$ packets, $\mathrm{CH}$ will derive an equation of the form $Y=a X+b$ where $a$ and $b$ are specific for each cluster member. Eventually, the cluster head sends all $a$ and $b$ to each cluster member.

As mentioned, Figure 5 shows that the convex hull technique can be used to derive lower and upper bounds on the local time of a remote node. In this case, each cluster head can generate a two dimensional graph for each cluster member after sending $m$ packets. The $\mathrm{x}$-axis and $y$-axis dimensions are local times of cluster member and cluster head repetitively. Thus, each cluster member has two clouds formed by lower-bound and upper-bond samples. Unlike liner regression that tends to average all the individual samples, this technique ignores average values and accounts for the samples with minimal or maximal error $[2,14]$.

Table 4 shows the number of messages used in the synchronization phase for SLTP, L-SYNC and L-SYNCng, $m, c$ and $n$ indicates number of synchronization packets , number of cluster heads and number of cluster members respectively. It is obvious that the linear regression

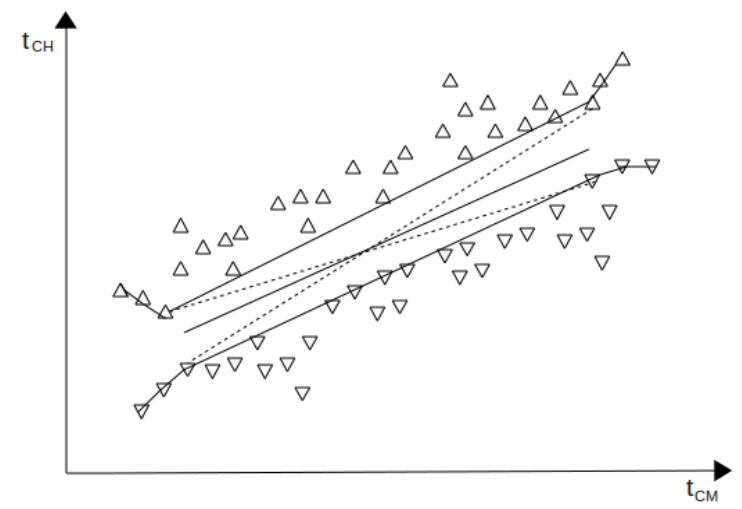

Figure 5. Convex Hull method.
Table 4. Number of transmitted messages for the following algorithms.

\begin{tabular}{cc}
\hline Algorithms & Number of messages \\
\hline SLTP & $\mathrm{C} * \mathrm{~m}$ \\
L-SYNC & $\mathrm{C} * \mathrm{~m}$ \\
L_SYNCng & $\mathrm{C} *[(1+\mathrm{n}) * \mathrm{~m}+\mathrm{n}]$ \\
\hline
\end{tabular}

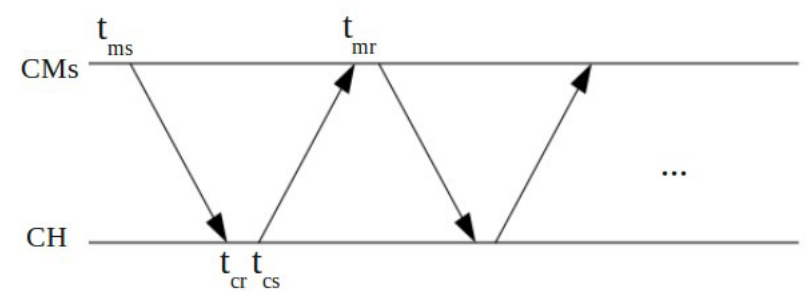

Figure 6. Synchronization packets between $\mathrm{CH}$ and CMs. CM starts synchronization.

used in SLTP and L-SYNC has fewer messages.

Another solution that can be discussed is to start sending synchronization packets by cluster members. Figure 6 shows this solution. With this solution, each cluster member sends $m$ synchronization packet to cluster head and then receives $m$ ACK messages. However, the number of messages in this method is $2 * m * n$ and has more overhead rather than first solution, although this solution has better scalability.

Between clusters, there are some nodes that receive timing packets from more than one group head; these are called gateways. Synchronization can be performed periodically at specific time intervals. But, if a node needs to be synchronized within these time intervals, it can broadcast a message for synchronization.

Any group head that receives this message will start to send its local time and ID. In the example shown in Figure 7, after executing the algorithm in cluster heads $\mathrm{CH} 1$ to $\mathrm{CH} 2$, nodes belonging to each group head will receive $a$ and $b$. If two nodes such as CM1 and CM2, that are the members of a cluster, want to communicate with each other, it is sufficient to send their $a$ and $b$ parameters to each other, and using the following equations they can convert their clocks. In a case where two nodes are not members of a cluster, they can also be synchronized. For instance, if nodes CM1 and CM3 want to be synchronized, it is enough to calculate $a$ and $b$

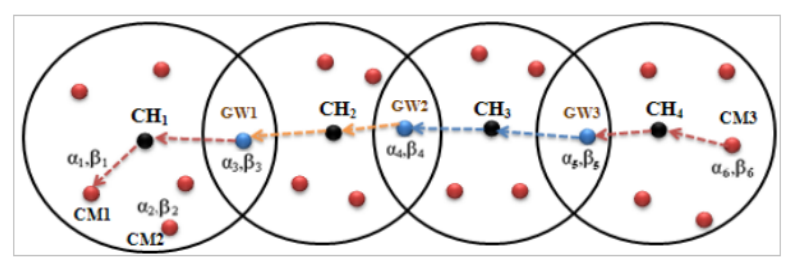

Figure 7. Synchronization of two nodes of two far clusters. 
parameters using Equation (2), in the path between each two nodes, in an appropriate route between nodes CM1 and CM3. We have proposed a routing algorithm between these nodes. Afterward, clock conversion will be done in each existing hop in the route path. Since conversion error in each hop will be added to the total error rate, the synchronization error increases with the number of hops. Consequently, clustering based on larger number of neighbors helps to shorten the route between two nodes, in order to perform fewer conversions and reduce the synchronization error rate.

$$
\begin{aligned}
& \mathrm{CM}_{1}: \mathrm{h}_{\mathrm{CH}_{1}}=a_{1} h_{\mathrm{CM}_{1}}+b_{1} \\
& \mathrm{CM}_{2}: \mathrm{h}_{\mathrm{CH}_{1}}=a_{2} h_{\mathrm{CM}_{2}}+b_{2}
\end{aligned} h_{\mathrm{CM}_{1}}=\frac{a_{2}}{a_{1}} h_{\mathrm{CM}_{2}}+\left(\frac{b_{2}-b_{1}}{a_{1}}\right)
$$

\section{Results}

To assess our synchronization protocol, we used the NS 2.31 simulator under the Linux operating system. We describe the simulation setup and configuration in detail in Subsection 5.1. Afterward, we selected SLTP [2] and L-SYNC [12] to compare our simulation results based on accuracy in terms of time and number of hops. We discuss our comparison in detail in Subsection 5.2.

\subsection{Simulation Setup}

To evaluate the proposed algorithm, it is required to consider several clocks, one for each node. To simulate several nodes' clocks on a system, a real time system was used, so that for each node's clock, one specific drift and one specific offset were selected.

Drift and offset are determined by a random function, between two identified values of max_drift and max_ offset, such that nodes' clocks are computed with the following equations. In our simulations, the worst cases for drift and offset have been considered. Simulation parameters are shown in Table 5.

When a node wants to be synchronized with another node, a packet is broadcast to form an optimized route between two nodes. In the return route, the required conversions are done. In each execution turn, different

Table 5. Simulation parameters values.

\begin{tabular}{lc}
\hline Subject & Value \\
\hline Number of synchronization packets & 10 \\
Time intervals between synchronization packets & $1 \mathrm{~s}$ \\
Synchronization period & $1000 \mathrm{~s}$ \\
Max. offset & $1 \mathrm{~s}$ \\
Max. drift & $0.0001 \mathrm{~s}$ \\
Simulation duration & $10262 \mathrm{~s}$ \\
\hline
\end{tabular}

routes between source and target will be selected, each experiment will be iterated 10 times and the results are averaged. Our simulation is done for two different topologies, heterogeneous and homogeneous. In homogeneous topology we use 100 nodes in $1000 * 1000$ square meters where each node has 100 meters range in a regular layout, and in heterogeneous topology the configuration is similar except that the nodes coordinates are randomly uniform.

$$
\text { time_node }=\text { current_time } * d r i f t+\text { offset }
$$

\subsection{Simulation Results and Comparison}

We simulated our work in two environments: noisy and noiseless. Each environment was tested with two topologies: homogeneous and heterogeneous. Figure 8 depicts the average error versus simulation time in noiseless homogeneous environment. It shows that as time passes the average error rate of L-SYNCng increases less than with the others.

Figure 9 depicts our second simulation in noiseless heterogeneous topology. It shows that as time passes the average error rate of L-SYNCng increases gradually, while for the other protocols it increases more rapidly.

We have repeated the two previous simulations innoisy environment where packets may arrive to destination with considerable delay. Figures 10-11 depict our simulation results. As we mentioned, in L-SYNC the protocol synchronization algorithm is based on linear regression technique. Linear regression can be influenced particularly by unreliable data which are far from the fitted line.

Figures 12-13 summarize L-SYNC and L-SYNCng general behavior in each different environment.

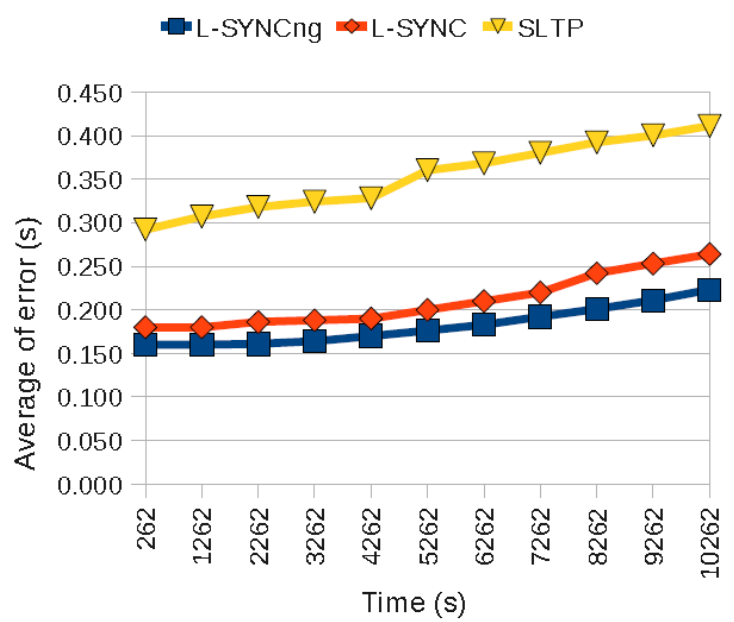

Figure 8. Comparison of L-SYNCng, L-SYNC and SLTP error versus time in homogeneous topology and noiseless environment. 


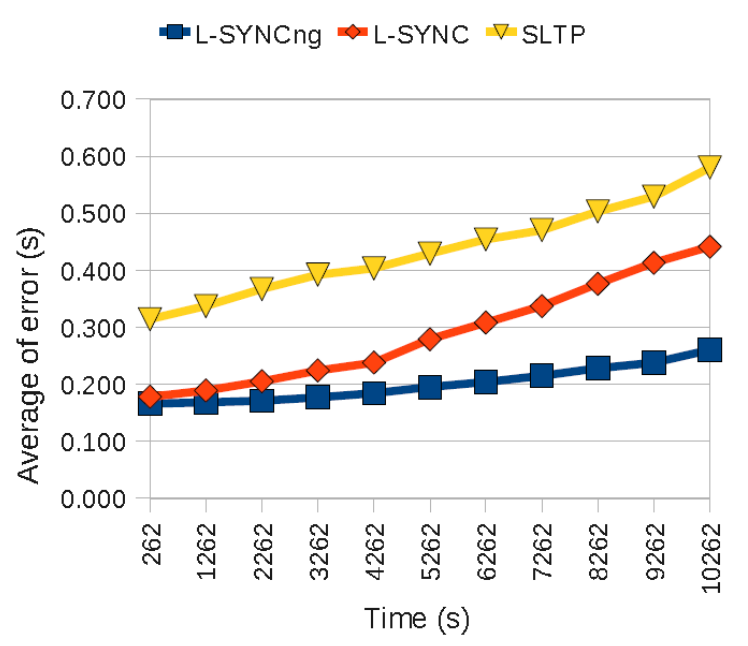

Figure 9. Comparison of L-SYNCng, L-SYNC and SLTP error versus time for heterogeneous topology and noiseless environment.

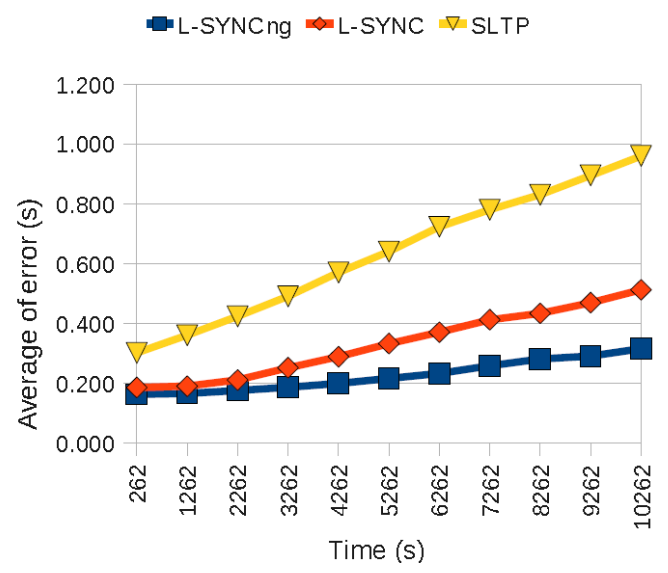

Figure 10. Comparison of L-SYNCng, L-SYNC and SLTP error versus time forhomogeneous topology and noisy environment.

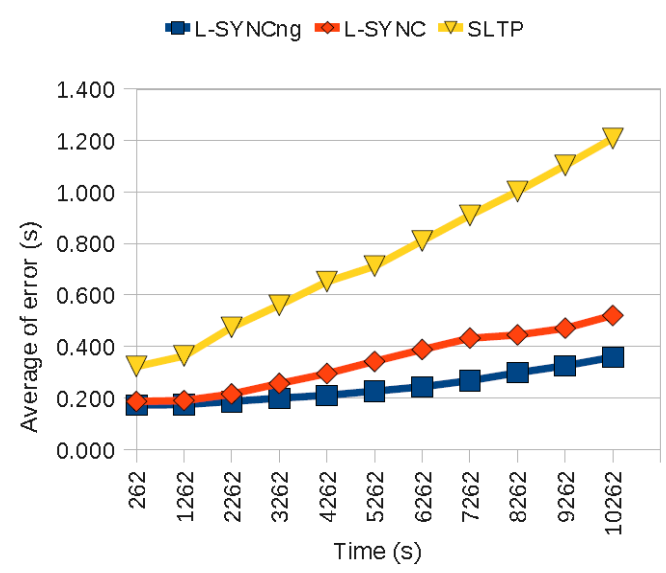

Figure 11. Comparison of L-SYNCng, L-SYNC and SLTP error versus time for heterogeneous topology and noisy environment.

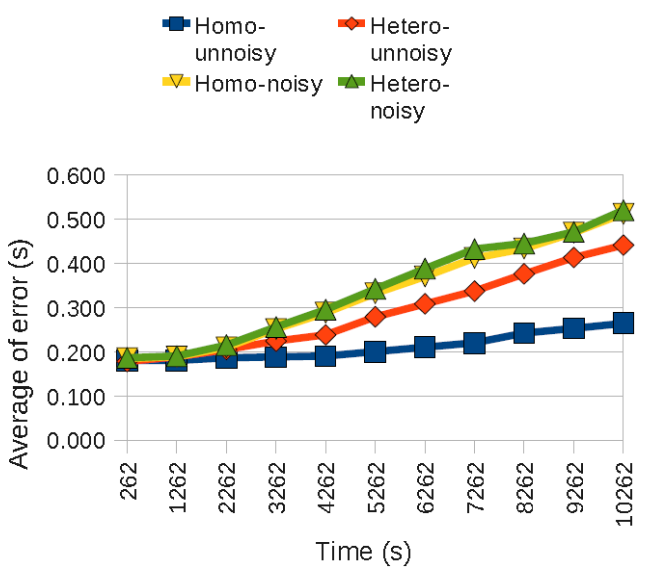

Figure 12. L-SYNCng behavior in different environment.

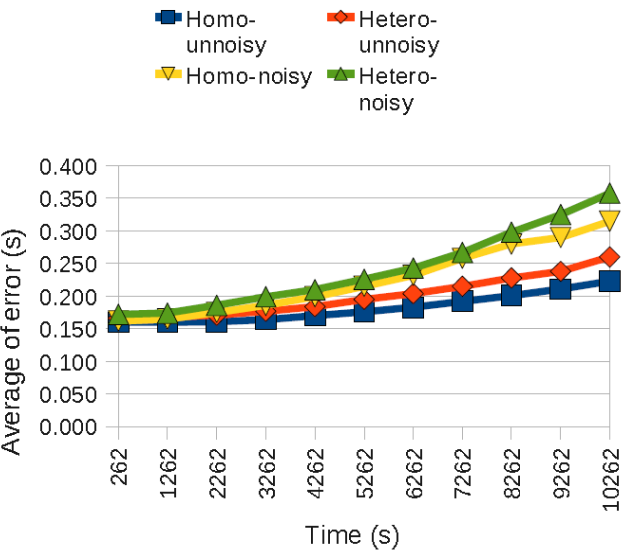

Figure 13. L-SYNC behavior in different environment.

\section{Conclusions and Future Work}

L-SYNCng is a reliable and efficient protocol for time synchronization in Wireless Sensor Networks. This protocol uses degree-based and weight-based clustering in heterogeneous and homogeneous topologies respectively. Using these clustering methods, L-SYNCng can reduce the number of hops in time synchronization process of two specific nodes which are in different clusters.

Moreover, L-SYNCng uses convex hull method to calculate clock offset and skew in each cluster. Therefore, it is capable to compute skew and offset intervals between each node and its corresponding head cluster. In other words, it can estimate the local time of remote nodes in the future and past. To estimate the local time for remote nodes, firstly a routing algorithm is used and afterward a conversion is performed in each hop. Simulation results illustrate that the convex hull method can increase efficiently the synchronization accuracy in noisy environments. As dynamic sensor networks might be indispensable in the future, we are going to apply LSYNCng to these environments where nodes change 
their status during time.

\section{References}

[1] H. Chan and A. Perrig, "ACE: An Emergent Algorithm for Highly Uniform Cluster Formation," Wireless Sensor Networks, Vol. 2920, January 2004, pp. 154171.

[2] www.vs.inf.ethz.ch/publ/papers/wsn-time-book.pdf

[3] J. Elson, L. Girod and D. Estrin, "Fine-Grained Network Time Synchronization, Using Reference Broadcasts," Proceedings of the 5th Symposium on Operating Systems Design and Implementation, Vol. 36, No. SI, Winter 2002, pp. 147-163.

[4] J. V. Greunen and J. Rabaey, "Lightweight Time Synchronization for Sensor Networks," Proceedings of the 2nd ACM International Conference on Wireless Sensor Networks and Applications, 2003, pp. 11-19.

[5] H. Dai and R. Han, “TSync: A Lightweight Bidirectional Time Synchronization Service for Wireless Sensor Networks," ACM SIGMOBILE Mobile Computing and Communications Review Archive, Vol. 8, No. 1, January 2004, pp. 125-139.

[6] S. Ganeriwal, R. Kumar and M. B. Srivastava, "Timing-Sync Protocol for Sensor Networks," Proceedings of the 1st International Conference on Embedded Networked Sensor Systems, 2003, pp. 138-149.

[7] Md. Mamun-Or-Rashid, C. S. Hong and C. Hyung, "Passive Cluster Based Clock Synchronization in Sensor Network," Advanced Industrial Conference on Telecommunications/Service Assurance with Partial and Intermittent Resources Conference/E-Learning on Telecommunications Workshop (AICT/SAPIR/ELETE'05), July 2005, pp. 340-345.

[8] H. Kim, D. Kim and S. Yoo, "Cluster-Based Hierarchical Time Synchronization for Multi-Hop Wireless Sensor Networks," 20th International Conference on Advanced Information Networking and Applications, Vol. 2, April 2006, pp. 318-322.
[9] K. Römer, "Time Synchronization and Localization in Sensor Networks,” Ph.D. Thesis, No. 16106, ETH Zurich, Switzerland, June 2005.

[10] M. Maroti, B. Kusy, G. Simon and A. Ledeczi, “The Flooding Time Synchronization Protocol," Proceedings of the 2nd International Conference on Embedded Networked Sensor Systems, SenSys, November 2004.

[11] S. N. Gelyan, A. N. Eghbali, L. Roustapoor, S. A. Yahyavi, F. Abadi and M. Dehghan, "Scalable Lightweight Time Synchronization, Protocol for Wireless Sensor Network," International Conference on Mobile Ad-Hoc and Sensor Networks, Beijing, December 2007, pp.536-547.

[12] M. Jabbarifar, A. S. Sendi, H. Pedram, M. Dehghan and M. Dagenais, "L-SYNC: Larger Degree Clustering Based Time-Synchronisation for Wireless Sensor Network," Eighth ACIS International Conference on Software Engineering Research, Management and Applications, Montreal, 2010.

[13] O. Younis, M. Krunz and S. Ramasubramanian, "Clustering in Wireless Sensor Networks: Recent Developments and Deployment Challenges," IEEE Network Magazine, Vol. 20, No. 3, May 2006, pp. 20-25.

[14] B. Poirier, R. Roy and M. Dagenais, “Accurate Offline Synchronization of Distributed Traces Using KernelLevel Events,” ACM SIGOPS Operating Systems Review, Vol. 44, No. 3, July 2010, pp. 75-87.

[15] A. Duda, G Harrus, Y. Haddad and G. Bernard, "Estimating Global Time in Distributed Systems,” Proceedings of the 7th International Conference on Distributed Computing Systems, Berlin, Vol. 18, 1987.

[16] S. Basagni, "Distributed Clustering Algorithm for Ad Hoc Networks," International Symposium on Parallel Architectures, Algorithms and Networks (ISPAN'99), 1999, pp. 310-315.

[17] L. M. Sichitiu and C. Veerarittiphan, "Simple, Accurate Time Synchronization for Wireless Sensor Networks," IEEE Wireless Communications and Networking Conference (WCNC’03), Vol. 2, March 2003, pp. 1266-1273. 